\title{
'|||||||||||||||||||||||||||||||||||||||||||||||||||||||||||||||||||||||||.
}

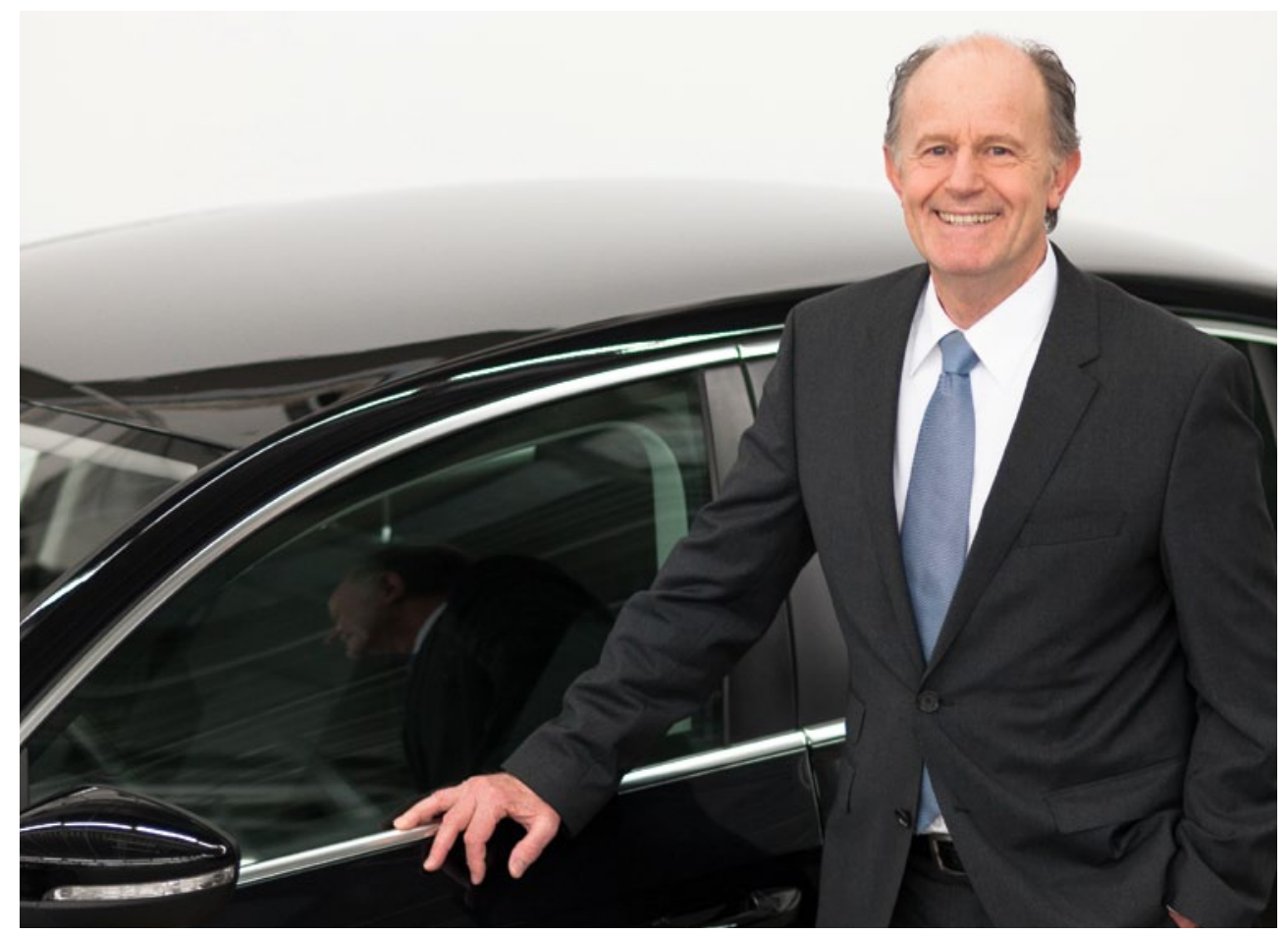

DR. PETER E. RIETH

Leiter Systems \& Technology, Mitglied der Geschäftsleitung, Division Chassis \& Safety, Continental

\section{ULTIMATIV ATTRAKTIV}

In den vergangenen Jahren haben sich Verkehrssicherheit und Fahrkomfort stetig erhöht. Maßgeblich mitverantwortlich für diesen positiven Trend sind Fahrerassistenzsysteme, die Unfälle vermeiden helfen und das Fahren angenehmer machen.

Der integrale Sicherheitsansatz ContiGuard von Continental leistet hierzu einen wesentlichen Beitrag. ContiGuard integriert aktive und passive Sicherheitssysteme, die durch Umfeldinformationen und Signalvernetzung effektiver zusammenwirken. Weitgehend standardisiert eingeführte Assistenzsysteme wie ABS, Airbags, Bremsassistent und ESC sorgen schon heute für ein schleuderfreies Fahren mit kurzen Bremswegen und bieten umfangreichen Insassenschutz. Auf dem Weg zum automatisierten Fahren spielen sie eine wichtige Rolle, da diesbezüglich neue Funktionen auf diesen Systemen aufbauen.

Bei fortschrittlichen Fahrerassistenzsystemen wie ACC, Kollisionswarnung, aktiver Notbremse oder Spurverlassenswarnung fährt der Fahrer noch selbst, wird aber situationsspezifisch bei der Bewältigung der Fahraufgabe unterstützt. Der Schritt vom assistierten zum automatisierten Fahren - vorerst für ausgewählte Fahraufgaben, beispielsweise das Staufahren - wird immer kleiner.

Die Assistenzfunktionen werden immer umfassender, das Bedienen des Autos durch den Fahrer tritt immer mehr in den Hintergrund. Zum Beispiel wird der Spurverlassenswarner zum teilautomatisierten Spurfahrassistenten. Das Assistenzsystem erledigt neben der Längsregelung nun auch die Querregelung, dem Fahrer obliegt die permanente Überwachungspflicht.

Der nächste große Schritt: Automatisiertes Fahren befreit den Fahrer (zeitweise) komplett von den Fahraufgaben (Bedienung und Überwachung) und schließt im konsequenten Schluss menschliches Fehlverhalten aus, welches zu annähernd $70 \%$ alleinige Ursache für Verkehrsunfälle ist.

Automatisiertes Fahren gibt dem Fahrer neue Handlungsfreiräume. Fahrtzeit ist nicht länger „verlorene Zeit“, sondern wird für ihn zu nützlicher Zeit, das Auto zum hoch attraktiven Verkehrsmittel. Die Akzeptanz des Fahrers wird es allerdings nur geben, wenn er sich sicher fühlt. Unfallfreies Fahren ist somit die unverzichtbare Voraussetzung für automatisiertes Fahren. Automatisiertes Fahren wird so zum Fortschrittsbeschleuniger für das Ziel unfallfreies Fahren. Um dieses Ziel zu realisieren, sind allerdings noch große Herausforderungen bei der Entwicklung im Bereich der Umfelderfassung und -interpretation, der Fahrzeuginformationsvernetzung, der Kontrolle der Fahrzeugbewegung sowie der Sicherheitsarchitektur im Hinblick auf die Ausfallsicherheit des Fahrzeugs zu bewältigen.

Die Fortschritte der Elektronik bei Rechenleistung, Speichergröße, Sensorik und Aktuatorik werden die Möglichkeit bieten, diese Herausforderung zu meistern. Elektronik kennt keine Schrecksekunde, sie ist immer hellwach. Fazit: Individuelle Mobilität der Zukunft - ultimativ attraktiv ... und ultimativ sicher. 